\title{
Diagnosis and treatment of Herlyn Werner Wunderlich syndrome: a case report
}

\begin{abstract}
Background: Herlyn Werner Wunderlich syndrome (HWWS) is a rare female urogenital tract abnormality, isolated by presenting a didelfous uterus, transverse vaginal septum and ipsilateral renal agenesis.

Objective: A case report of a patient with Werner Herlyn Wunderlich syndrome.

Case report: Female patient of 17 years of age, single, nubile, menarche at 14 years, infrequent menstrual cycles, normal volume, with dysmenorrhea EVA 7/10, attends to present disabling pelvic pain, ultrasound was found finding, hematocolpos and hematometra, is complemented with hysteroscopy, computed tomography (CT) and magnetic resonance imaging (MRI), a diagnosis of HWWS syndrome is integrated by confirming the presence of a didelfo uterus, obstructed right hemivagina and right renal agenesis, with distention of the vagina and cervix secondary to hematometra and hematocolpos, normal ovaries. The approach in this patient was by minimal invasion.
\end{abstract}

Conclusion: Herlyn Werner Wunderlich syndrome is a rare mullerian defect, it is often diagnosed late, the diagnosis is usually made after menarche, associated with chronic pelvic pain or palpable pelvic mass; 2D ultrasound along with physical examination performs the suspected diagnosis; This should be complemented with RM. The treatment of choice is vaginal septum resection assisted by minimally invasive techniques.

Keywords: herlyn werner wunderlich syndrome, uterus didelphys, renal agenesis
Volume II Issue I - 2020

\author{
Rubén Hernández Silva Jesus,' Gabriel \\ Garcia Gerardo Hernandez, ${ }^{2}$ José Pérez \\ López El Carmen, ${ }^{3}$ Clara Magdalena \\ Martínez Hernández, ${ }^{4}$ Roberto Pablo \\ Gonzalez Mendez, ${ }^{5}$ Patricia Lopez Ramirez ${ }^{6}$ \\ 'Medical resident $4^{\circ}$ gynecology and obstetrics, HRAEM, Mexico \\ ${ }^{2} \mathrm{Head}$ of gynecology, HRAEM, Mexico \\ ${ }^{3}$ Gynecology attending physician, HRAEM, Mexico \\ ${ }^{4} \mathrm{Head}$ of research, HRAEM, Mexico \\ ${ }^{5}$ Gynecology attending physician, IMSS No. 12, Mexico \\ ${ }^{6}$ Resident physician, 4th gynecology and obstetrics, HRARM, \\ Mexico
}

Correspondence: Dr. Ruben Jesús Hernández Silva, Medical Resident, $4^{\circ}$ gynecology and obstetrics, HRAEM, Regional High Specialty Hospital of Women Villahermosa, Tabasco, Mexico,Email jesus_ruben9@hotmail.com

Received: December 26, 2019 | Published: February 10, 2020
Abbreviations: HWWS, Herlyn Werner Wunderlich syndrome; MRI, magnetic resonance imaging; CT, computed tomography; OHVIRA, hemivagina Obstructed and ipsilateral renal anomaly; ESHRE/ESGE, European Society of Human Reproduction and Embryology/European Society of Gynecologic Endoscopy

\section{Background}

The Mullerian alterations represent a series of alterations in the uterine anatomy as a result of the poor development of the Mullerian ducts. ${ }^{1}$

From Müllerian duct fallopian tubes, uterus, cervix and upper vagina $2 / 3$ develop. $^{2}$

Herlyn Werner syndrome Wunderlich is an abnormality of the rare female genital tract which is characterized by a uterus didelphic hemivagina obstructed by a transverse vaginal septum and renal agenesis. Also called syndrome OHVIRA (hemivagina Obstructed and ipsilateral renal anomaly). ${ }^{3}$

The incidence of the syndrome Werner syndrome Herlyn Wunderlich not known accurately, it is estimated at 1: 6000 to 1: 28,000 cases of Mullerian malformations or $6 \%$ of patients with didelphic uterus. ${ }^{4}$

HWW syndrome is induced Mullerian anomaly by damage to the duct which originate Wolff ipsilateral kidneys and induce adequate Müllerian duct in its flow portion, in the eighth week of gestation fusion. If the duct Wolff is absent, then the Mullerian duct is positioned laterally incorrectly. As a result of the move away from the midline, no fuses with the contralateral duct causing a didelphic uterus and not merged with the urogenital sinus centrally. The conduit not displaced form the vagina that connects to the outside while the displaced conduit form a blind pouch. ${ }^{5}$

Commonly diagnosed after menarche due to pelvic and abdominal pain. Often the diagnosis is late, which increases the risk of complications such as endometriosis, hematocolpos, hematometra and infertility. ${ }^{3}$ Ultrasound diagnostic suspicion and for preoperative evaluation is performed is complemented by CT or MRI; the latter is considered the gold standard (Figure 1) (Figure 2). ${ }^{6}$

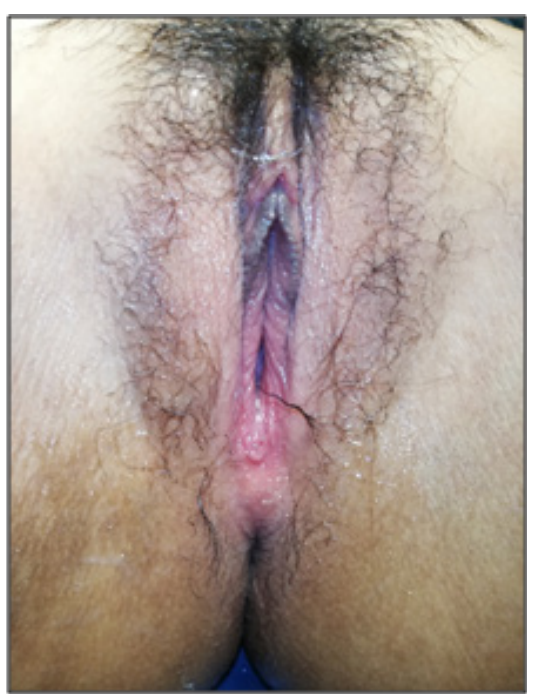

Figure I External Genital normal. 


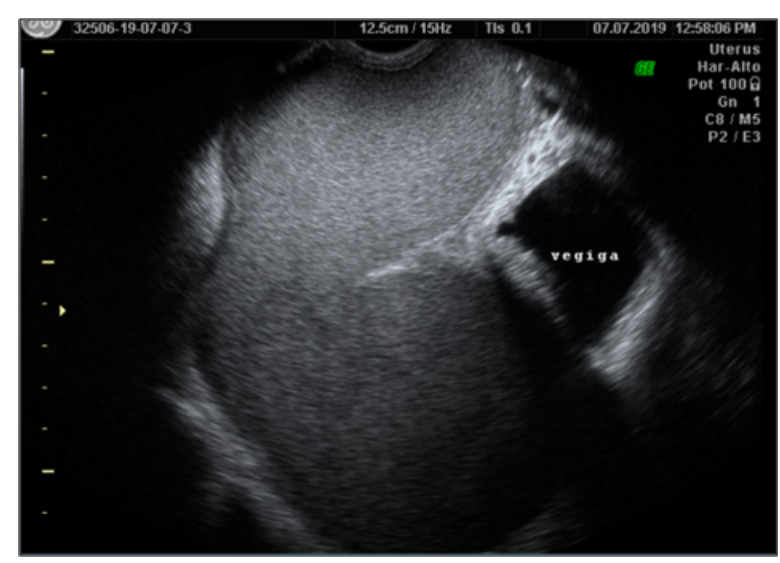

Figure 2 Hematometra and hematocolpos of hemiuterus and right hemivagina.

\section{Clinical case}

MRI and CT diagnostic didelphic corroborates uterus with right renal agenesis (Figure 3), loosening of vagina and cervix secondary to hematometra and hematocolpos, normal ovaries. Hysteroscopy fails to go right cavity to present transverse vaginal septum, convex, left cavity with eutrophic cervix, endocervical canal, cavity and normal ipsilateral left ostium (Figure 4).

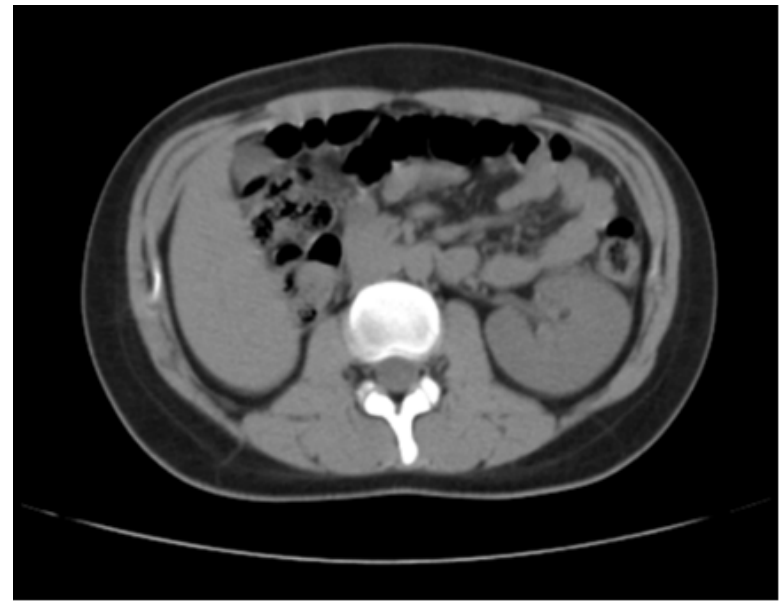

Figure 3 TC, kidney normal left and right renal agenesis.

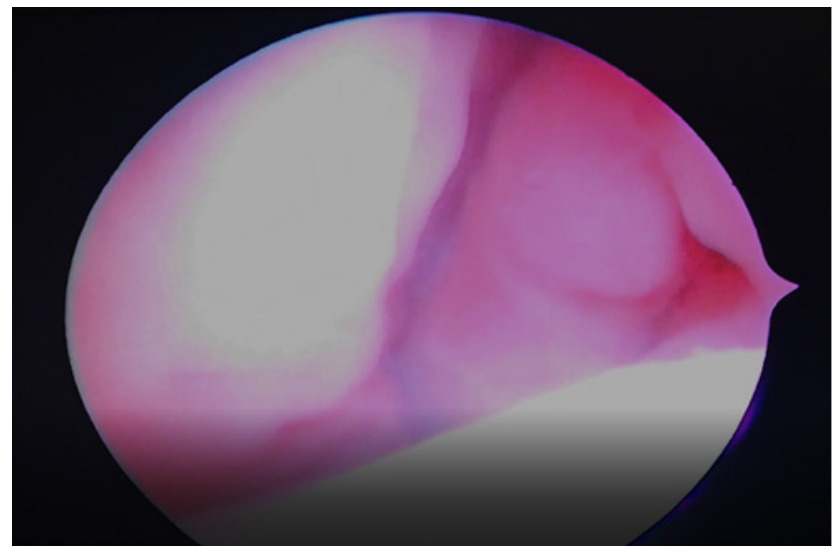

Figure 4 Cervix left normal vaginal wall bulging right.
Balanced laparoscopy under general anesthesia was performed by examining pelvic organs where didelphic seen uterus entitled hemiuterus increased volume, endometriotic red, hematosalpinx and salpinge right attached to the ovary. Electrofulguration of endometriotic foci is performed with bipolar energy and salpingoovariolisis. Longitudinal $1 \mathrm{~cm}$ incision at the level of insertion of the uterosacral ligaments right hemiuterus monopolar energy is introduced clamp grasper and used as a guide to locate the ideal site section vaginal septum under direct vision incision vaginal septum is made in it transverse direction with a length of $2 \mathrm{~cm}$, with vicyl marzupializa 00 (Figure 5). Closure in two planes with vicryl of 1 backside uterine.

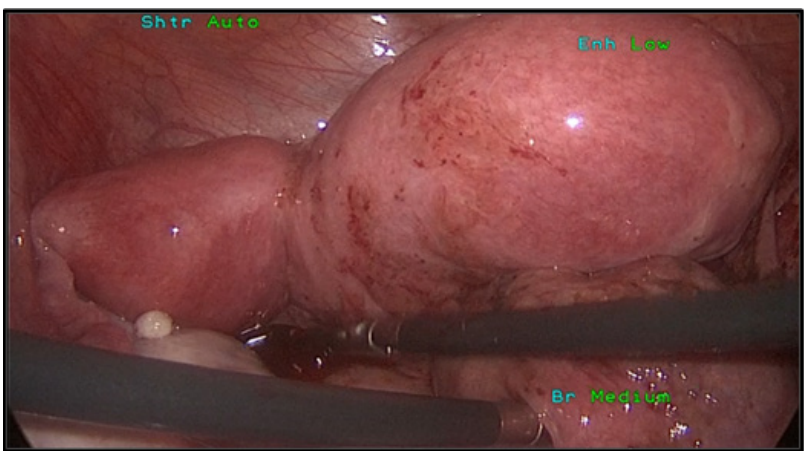

Figure $\mathbf{5}$ Uterus didelphic with endometriotic, hematómetra hemiuterus law.

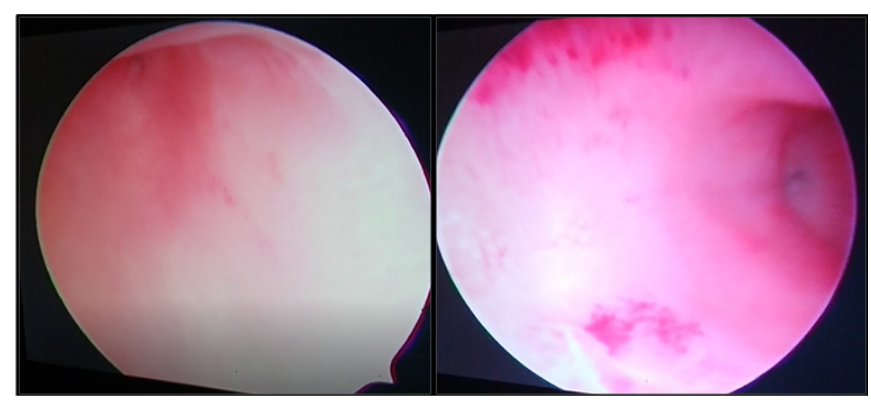

Figure 6 hysteroscopy; observing the two uterine cavities and their respective.

Adequate postoperative course, withdrawing the service at 48 hours, monitoring the out patient gynecology, without presenting clinical picture recurrence, performing hysteroscopy control at 3 months cervix and achieving the right to observe normal uterine cavity.

\section{Discussion}

HWW syndrome is a very rare Mullerian defect. It occurs around the ninth week of pregnancy, by a failure in the fusion of the Mullerian ducts vertically and laterally. Its etiology is unknown and is characterized by the triad of didelphic uterus, obstructed hemivagina and unilateral renal agenesis. ${ }^{4}$

The clinical picture depends on the degree Mullerian altereacion. Diagnosis may occur as clinical symptoms in patients in the study of infertility or as an incidental finding. ${ }^{7}$

In this case the diagnosis was made later menarche, with the cardinal symptom chronic pelvic pain, which occurs in up to $90 \%$ of patients; Retrograde menstruation associated and complicated with hematocolpos, hematómetra and hematosalpinx. ${ }^{3}$ 
Tong and his group reported an incidence of $17 \%$ and endometriosis Fedele Land collaborators $13.8 \%$, in our case associated with endometriosis was reported. ${ }^{8}$

According to the classification proposed by Lan Zhu et al. ${ }^{9}$ A syndrome the classified into two types.; Classification 1 with complete obstruction classification hemivagina and 2 with partial obstruction. These in turn are subdivided into 2. Classification 1.1 as in our case hemivagina is completely blocked, the uterus is no communication to contralateral side. Classification 1.2 hemivagina completely obstructed with atresia of the cervix. 2.1 classification there is little communication between the two hemivaginas through an incomplete communication of the vaginal septum. 2.2 Classification hemivagina completely obstructed with partial communication between the two cervix. ${ }^{9}$

According to the classification of the European Society of Human Reproduction and Embryology/European Society of Gynecologic Endoscopy.

(ESHRE/ESGE) (2013) HWW syndrome is in the class U3B uterine abnormality, $\mathrm{C} 2$ and $\mathrm{V} 3$ vaginal cervical abnormality anomaly. ${ }^{6}$

In this case the suspected diagnosis is performed with ultrasound, identifying hematocolpos, hematómetra and Mullerian defect confirming the diagnosis with hysteroscopy, computed tomography and magnetic resonance; the latter considered the gold standard and essential for establishing a surgical plan., ${ }^{9,10}$

The ipsilateral renal agenesis presented here as is reported in 92 to $100 \%$ of patients with obstructed hemivagina didelphic uteri. ${ }^{4}$ However, renal duplication and multicystic dysplastic kidney have also been reported.

Minimally invasive surgery for these patients is choice, in this case the approach and hysteroscopy laparoscopy was performed with resection of the transverse vaginal septum, electrofulguration of endometriotic foci and salpingoovariolisis. ${ }^{5,10-12}$

\section{Acknowledgments}

None.

\section{Funding}

None.

\section{Conflicts of interest}

The authors declare there are no conflicts of interest.

\section{References}

1. Sadek SM, Ahmad RA, Atia H. Performance of the ESHRE/ESGE classification in differentiating anomalies of double uterine cavity in comparison with the ASRM classification. Middle East Fertil $J$. 2016;21(2):75-81

2. Chandrayan P, U Parekh, Jain N. Mullerian duct anomalies presenting with primary amenorrhoea. Int J Reprod Contraception, Obstet Gynecol. 2016;5(2):300-305.

3. BRE Ruz, Leroy LL, Gallardo VLE, et al. Laparoscopic approach syndrome Herlyn Werner Wunderlich. 2015;13(4):259-262.

4. Huneeus A, AS Acuna, Manuel V, et al. Clinical cases syndrome hemivagina obstructed, uterus didelphic and ipsilateral renal agenesis (OVHIRA) drained partly by fistula uterus-uterine. Rev Chil Obstet Gynecol. 2017;82(6):595-602.

5. Kriplani A, V Dalal, Kachhawa G, et al. minimally invasive endoscopic approach for management of OHVIRA syndrome. J Obstet Gynecol India. 2019;69(4):350-355.

6. Heinonen PK. Distribution of female genital tract anomalies in two classifications. Eur J Obstet Gynecol Reprod Biol. 2016;206:141-146.

7. Rezai S, L Angeles, Hughes A, et al. Three cases of didelphys uterus, including uterus didelphys obstructed with renal agenesis hemivagina and ipsilateral (OHVIRA Syndrome) AKA Herlyn- Werner-Syndrome Wunderlich, with a systematic review. Obstet Gynecol Int J. 2017;7(2):00241.

8. Sepúlveda-Agudelo J, Jaimes-carvajal H. Herlyn-Werner-Wunderlich Syndrome: of the different types of manifestation. Report of two cases Gynecol Obstet Mex. 2019;87(2):139-145.

9. Zhu L, Chen N, Tong J, et al. New classification of Herlyn-WernerWunderlich syndrome. Chin Med J. 2015;128(2):222-225.

10. Senger K, Singh A, Ravikumar R, et al. Detection of mullerian duct anomalies: diagnostic utility of two dimensional ultrasonography as to compared magnetic resonance imaging. Int J Reprod Contraception, Obstet Gynecol. 2017;6(1):20-28.

11. Poly-Neto OB, Rosa-e-Silva JC, Nogueira AA, et al. Diagnosis and treatment in a tertiary hospital of a series of complex genital malformations corresponding to double uterus with obstructed hemivagina and ipsilateral renal agenesis. Int J Reprod Med. 2018.

12. Plans C, Lopez E, Lopez MJ, et al. Herlyn-Werner SyndromeWunderlich. Gin Obst Clin Invest. 2015;42(4):186-188. 